\title{
BMJ Open Levosimendan and Global Longitudinal Strain Assessment in Sepsis (GLASSES 1): a study protocol for an observational study
}

\author{
lacopo Cappellini (D) , Alessandra Melai, ${ }^{1}$ Lucia Zamidei, ${ }^{1}$ Maddalena Parise, ${ }^{1}$ \\ Simone Cipani, ${ }^{2}$ Guglielmo Consales ${ }^{1}$
}

To cite: Cappellini I, Melai A, Zamidei L, et al. Levosimendan and Global Longitudinal Strain Assessment in Sepsis (GLASSES 1): a study protocol for an observational study. BMJ Open 2020;10:e037188. doi:10.1136/ bmjopen-2020-037188

- Prepublication history for this paper is available online. To view these files, please visit the journal online (http://dx.doi org/10.1136/bmjopen-2020037188).

Received 22 January 2020 Revised 03 August 2020 Accepted 07 August 2020
Check for updates

(C) Author(s) (or their employer(s)) 2020. Re-use permitted under CC BY-NC. No commercial re-use. See rights and permissions. Published by BMJ.

${ }^{1}$ Department of Critical Care Section of Anesthesiology and Intensive Care, Azienda USL Toscana Centro, Prato, Italy ${ }^{2}$ Department of Critical Care Section of Anesthesiology and Intensive Care, Azienda USL Toscana Centro, Figline Valdarno, Italy

Correspondence to Dr lacopo Cappellini; iacopo.cappellini@usıcentro. toscana.it

\section{ABSTRACT}

Introduction Cardiogenic shock is a condition of low cardiac output that represents the end stage of a progressive deterioration of cardiac function. The main cause is ischaemic heart disease, but there are several non-ischaemic causes, including sepsis. The use of levosimendan in cardiogenic shock during sepsis is still under debate.

Methods We are conducting an observational, singlecentre, not-for-profit study enrolling patients aged 18-80 years old admitted to the intensive care unit with a diagnosis of septic shock. Patients will be monitored with the EV1000/VolumeView device (Edwards Lifesciences, Irvine, USA). Patients with cardiac index $(\mathrm{Cl})$ values $<2.5 \mathrm{~L} /$ $\mathrm{min} / \mathrm{m}^{2}$ and/or stroke volume index (SVI) $<30 \mathrm{~mL} /$ beat $/ \mathrm{m}^{2}$ are considered eligible for the study. Enrolled participants will undergo an echocardiographic examination using the Vivid S6 ultrasound machine (General Electric, Northville, Michigan) and a 3.6 MHz cardiology probe through which the apical projections of chambers 2, 3 and 4 will be acquired; this is necessary to calculate the global longitudinal strain (GLS) using EchoPAC* Clinical Workstation Software (General Electric). A dobutamine infusion will be started in these patients; 24 hours later $\mathrm{Cl}$ and SVI will be recalculated using EV1000/NolumeView and then a levosimendan infusion will begin for 24 hours. Once the infusion cycle of the calcium-sensitising drug has been carried out, the infusion of dobutamine will be reduced until it stops, and the $\mathrm{Cl}, \mathrm{SVI}, \mathrm{GLS}$ and arterial elastance (Ea):Ventricular Elastance (Ees) will be reevaluated. The primary endpoint is recovery of GLS $\geq 15 \%$ and the secondary endpoint is a relative reduction in mortality of $15 \%$.

Ethics and dissemination The investigators declare that the study will be conducted in full compliance with international regulations (EU Directive 2016/679/ EC) and national implementation (DM 15 July 1997; $211 / 2003 ; 200 / 2007$ ) regarding the clinical trial and the principles of the Declaration of Helsinki. Study results will be disseminated through peer-reviewed journals and conferences. Ethical approval for this study has been given by Comitato Etico Regione Toscana - Area Vasta Centro, Florence, Italy (ethical committee number: 13875_oss) on 25 May 2019 (Chairperson Professor Marco Marchi). Trial registration number NCT04141410.
Strengths and limitations of this study

- The present study does not include patients with ischaemic heart disease, which could affect response to inotropic drugs.

- Global longitudinal strain, a prognostic factor of mortality, was used in order to understand if inotropic drugs could be beneficial in cardiogenic shock during sepsis.

- The use of a system of haemodynamic monitoring is important in order to evaluate improvements in cardiac contractility and cardiac index.

- This is a single-centre study.

- This an observational study without randomisation of treatment into two or more groups.

\section{INTRODUCTION}

Cardiogenic shock is a condition of low cardiac output that represents the end stage of a progressive deterioration of cardiac function. The main cause is ischaemic heart disease, but there are several non-ischaemic causes, including sepsis. ${ }^{1}$

Sepsis is characterised by organ dysfunction caused by an altered response of the body to an infection. Organ dysfunction can be defined as a change in Sequential Organ Failure Assessment (SOFA) score $\geq 2$, which reflects a mortality risk of roughly $10 \%$. Its most severe form is septic shock, defined as sepsis with hypotension unresponsive to fluid administration, necessitating vasoactive drugs, and with serum lactate $\geq 2 \mathrm{mmol} / \mathrm{L}$. With the occurrence of this condition, mortality rate increases to almost $40 \%{ }^{2}$

Cardiac dysfunction in sepsis is characterised by low cardiac output not related to myocardial ischaemia. ${ }^{3}$

Two-dimensional transthoracic echocardiography is currently one of the first investigations to be conducted on patients with cardiogenic shock. It can be carried out 
quickly as a non-invasive tool. During sepsis the parameters conventionally used for global cardiac evaluation, such as the ejection fraction, may not be suitable for diagnosis given the huge variability of preload and afterload. ${ }^{45}$ Other echocardiographic parameters have been used to assess cardiogenic shock in sepsis, such as the myocardial performance index (or the Tei index), tissue Doppler estimation or afterload-adjusted cardiac performance. However, the evaluation of these parameters may be strictly dependent on the evaluation angle used and is less faithfully reproducible. ${ }^{6}$

Speckle tracking echocardiography (STE) was first described in 2004 as a non-Doppler, angle-independent method for estimating left ventricular function. It uses a semiautomatic algorithm that tracks the displacement of acoustic speckles in the myocardium, evaluating the length variation of the myocardial segments. By directly measuring myocardial deformation, STE is less dependent on other parameters such as load changes and myocardial compliance. The most common parameter measured with STE is the 'strain', defined as the variation in length of myocardial fibres at the end of a systole compared with their original length at the end of a diastole, expressed as a percentage. The strain can be measured in longitudinal, radial and circumferential directions. The global longitudinal strain (GLS) is the mean of the longitudinal strain measured in 17 myocardial segments and has been validated as the most reproducible measurement. ${ }^{7}$ The American Heart Association estimates that the GLS of a healthy heart is about 20\%. ${ }^{8}$ A 2015 initiative involving the European Association of Cardiovascular Imaging, the American Society of Echocardiography and the manufacturers of ultrasound instruments confirmed the validity, reproducibility and superiority of the estimate of myocardial contractility obtained with speckle tracking compared with the best known echocardiographic parameters. ${ }^{6}$ The estimate of longitudinal tension has also proved to be a valuable tool for early detection of signs of initial myocardial damage and to correlate very well with the measurements obtained with MRI, considered the gold standard in the evaluation of cardiac contractility.

In the Early Goal Directed Therapy (EGDT) of the Surviving Sepsis Campaign, the use of dobutamine is recommended as a first-choice drug in patients with cardiogenic shock. The use of alternative inotropes is also mentioned, particularly levosimendan, without however highlighting differences in terms of mortality compared with dobutamine or a placebo. ${ }^{9}$ In three recent randomised trials (ProCESS, ProMISe and ARISE), the use of dobutamine according to EGDT did not result in differences in mortality compared with patients who did not receive it, as if its use had been overemphasised against a non-significant effect on mortality in a patient with sepsis. In addition, it was found that the use of this inotrope is associated with increased mortality in a patient with cardiogenic shock during sepsis. Indeed, with an increase in the cardiac index, dobutamine also causes an increase in the oxygen demand of myocytes, thus increasing the risk of ischaemia and tachyarrhythmias. The effects of dobutamine in a patient with sepsis are therefore unclear and may even be harmful for patients. Conversely, the use of beta-blockers in septic shock has been linked to an increase in survival rate in some studies. ${ }^{10}$

The use of levosimendan in cardiogenic shock during sepsis was first described in a 2005 case report. ${ }^{11}$ Since then there have been small studies and other case reports that have shown better outcomes in terms of right and left ventricular contractility, ventricular coupling, cardiopulmonary performance, global oxygen and splanchnic perfusion when compared with dobutamine and placebo. ${ }^{12}{ }^{13}$ Over time, other beneficial effects of this drug have emerged, including an anti-inflammatory, antioxidant and antiapoptotic action with possible protection from ischaemia-reperfusion damage. ${ }^{14-16}$

In the literature data on the use of levosimendan as an inotrope in patients with septic shock are encouraging but come from few studies on small samples. The LeoPARDS (Levosimendan for the Prevention of Acute Organ Dysfunction in Sepsis) trial on the use of levosimendan in septic shock has too many limitations to justify excluding this drug from therapy in sepsis; above all the inaccurate assessment of cardiac function did not permit an appropriate use of the drug as a means for weaning from inotropic drugs in continuous infusion. Although it is evident that STE and arterial ventricular coupling permit the diagnosis of cardiogenic shock with relative precision, there are no studies that use this type of echocardiographic evaluation to evaluate the effects of inotropes, and in particular of levosimendan, on the myocardium affected by cardiac dysfunction during sepsis. The present study aims to evaluate the correct use of levosimendan in cardiogenic shock with low cardiac index to wean patients off inotropic drugs in infusion.

\section{METHODS AND ANALYSIS}

The present study is an observational, single-centre, notfor-profit study. The expected duration is 12 months, starting from September 2019. Ethical approval for this study has been given by Comitato Etico Regione Toscana - Area Vasta Centro, Florence, Italy (ethical committee number: 13875_oss) on 25 May 2019 (Chairperson Professor Marco Marchi).

The present study will be carried out in the intensive care unit of the Santo Stefano Hospital in Prato, Italy, between September 2019 and September 2020, with a follow-up at 28 and 90 days after discharge from the intensive care unit.

All consecutive patients with the following inclusion criteria will be enrolled in the study:

- Informed consent.

- Age between 18 and 80 years old.

- Diagnosis of sepsis according to the Third International Consensus Definitions for Sepsis and Septic Shock. ${ }^{2}$ 
- Diagnosis of cardiogenic shock with heart index $<2.5 \mathrm{~L} / \mathrm{min} / \mathrm{m}^{2}$, calculated by EV1000/VolumeView thermodilution method, and/or diagnosis of stroke cardiogenic shock volume index $<30 \mathrm{~mL} /$ beat $/ \mathrm{m}^{2}$, calculated by EV1000/VolumeView thermodilution method.

- Need for dobutamine and levosimendan according to the standard procedure of current clinical practice at the centre.

Patients with the following exclusion criteria will not be enrolled:

- Age $<18$ years and $>80$ years.

- Pre-existing diagnosis of heart failure with a reduced or preserved ejection fraction.

- History of valvular heart disease or valve replacement and/or placement of cardiac implantable devices.

- Severe pulmonary hypertension and cor pulmonale.

- Poor acoustic windows.

- History of hypersensitivity or allergy to levosimendan. Each participant will undergo echocardiographic examination with a Vivid S6 ultrasound machine (General Electric, Northville, Michigan) and a $3.6 \mathrm{MHz}$ cardiology probe through chambers 2, 3 and 4; apical projections will be acquired in order to calculate the GLS using EchoPAC* Clinical Workstation Software (General Electric).

During the echocardiographic examination, the Ea:Ees ratio (ventricular-arterial coupling) will also be calculated using the method described by Chen et $\mathrm{l}^{17}$ modified on a single beat ( $\mathrm{t} 0$ ) using the iElastance application. Patients will be monitored with the EV1000/VolumeView device (Edwards Lifesciences, Irvine, USA), which measures the cardiac index (CI) and the stroke volume index (SVI).
Patients with Ea:Ees $>1$, CI values $<2.5 \mathrm{~L} / \mathrm{min} / \mathrm{m}^{2}$ and/ or SVI $<30 \mathrm{~mL} /$ beat $/ \mathrm{m}^{2}$ will be considered eligible for enrolment. In these patients, dobutamine infusion will be started from $5 \mu \mathrm{g} / \mathrm{kg} / \mathrm{min}$ following the bundles of the Surviving Sepsis Campaign at dosages where it is possible to obtain an $\mathrm{CI}>2.5 \mathrm{~L} / \mathrm{min} / \mathrm{m}^{2}$ and $/$ or SVI $>30 \mathrm{~mL} /$ beat/ $\mathrm{m}^{2}$. Moreover, if the systemic vascular resistance index is $<1200 \mathrm{dyn} * \mathrm{~s}^{*} \mathrm{~cm}^{-5}$, an infusion of norepinephrine will be started in order to maintain a mean arterial pressure (MAP) of above $65 \mathrm{~mm} \mathrm{Hg}$; if high dosage $(>0.5 \mu \mathrm{g} / \mathrm{kg}$ / $\min$ ) of norepinephrine is not sufficient to obtain the target of blood pressure, an infusion of vasopressin will be added, as suggested by the Surviving Sepsis Campaign. ${ }^{9}$ After 24 hours of dobutamine infusion, the CI and SVI will be recalculated using EV1000/VolumeView, GLS and the ventricular-arterial coupling index by ultrasound investigation; a 24-hour levosimendan infusion will begin with a starting dose of $0.1 \mu \mathrm{g} / \mathrm{kg} / \mathrm{min}$ in order to wean the patient off dobutamine. Once the infusion cycle of the calcium-sensitising drug has been carried out, the infusion of dobutamine will be reduced if possible until it stops, and the CI, SVI, GLS and Ea:Ees will be re-evaluated. The same echocardiographic evaluations and haemodynamic calculations using EV1000/VolumeView will be performed 72 and 96 hours after the start of the dobutamine infusion (figure 1). All patients enrolled will be followed up by the Medical Outcomes Survey ShortForm 36 (SF-36) questionnaire 28 and 90 days after discharge from intensive care.

It should be noted that all the procedures described (including echocardiographic monitoring) comply in quantity and frequency with the normal standard of care and management of patients admitted to intensive care

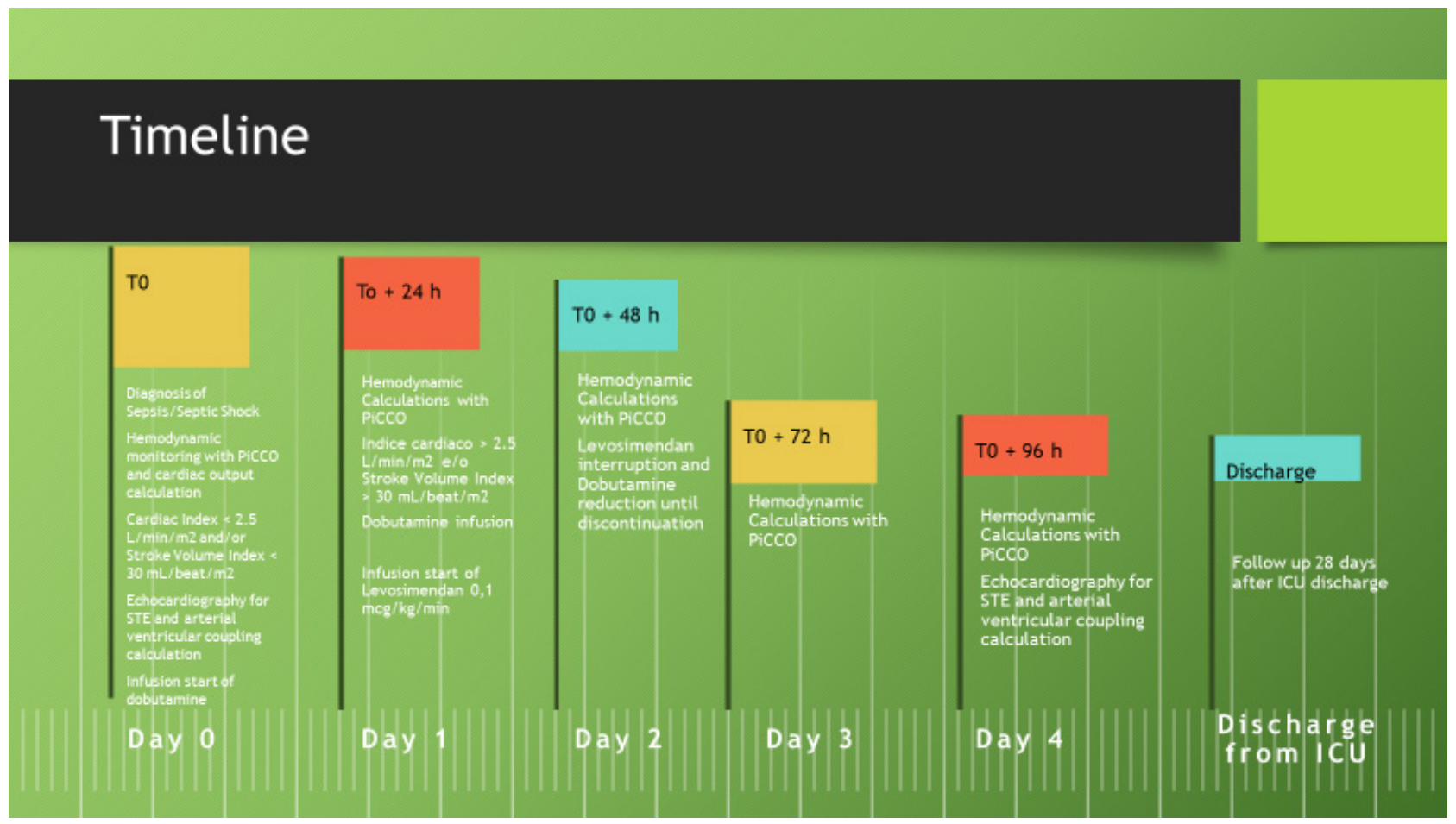

Figure 1 Timeline of the study. 
with a diagnosis of cardiogenic shock during sepsis, at Azienda USL Toscana Centro.

In addition, the parameters measured during the echocardiographic examination are derived from the reprocessing of the images collected during the examination and therefore simply represent an indepth examination for diagnostic purposes, performed according to current practice at the centre.

\section{Primary endpoint}

The primary endpoint is recovery of normal values of GLS $\geq 15 \%$ after infusion of dobutamine and levosimendan.

\section{Secondary endpoint}

The secondary endpoint is that participants with GLS $>15 \%$ will have $30 \%$ lower mortality rate than patients with GLS $<15 \%$.

\section{Variables}

Data will be described according to demographic characteristics, clinical parameters at baseline, and clinical parameters of efficacy and safety.

For continuous variables, the parameters of central tendency and dispersion such as arithmetic mean, SD, minimum, maximum, median, and first and third quartiles will be reported, while for categorical variables (both nominal and ordinal) the analyses on absolute and relative frequencies will be reported.

\section{Bias}

In order to minimise or avoid systematic errors, the data will be collected by qualified observers, prepared according to the guidelines of Good Clinical Practice (GCP), who will not participate in the clinical care of the patient. ${ }^{1819}$ Data input within the case report form (CRF) will be double-checked by experienced staff. In addition, the sample selection includes the introduction of objective selection criteria in order to minimise sampling biases in the study.

\section{Sample size}

A sample size of 63 patients has a power of $80 \%$ (calculated using the normal approximation method) to detect a difference of 0.15 in the proportion of responders between the null and the alternative hypothesis, using an exact two-tailed test with a level of significance (alpha) of 0.05 . These results assume that the proportion of responders under the null hypothesis is 0.8 (calculation performed with PASS V.15, NCSS V.15.0.2 2017). Considering a possible dropout rate of about $15 \%$, the final sample size necessary is 74 patients.

A responder is a patient who presents on day 2 (from the start of the infusion) with a $50 \%$ reduction of the administered dobutamine with respect to the initial infusion.

\section{Enrolment procedure}

In order to promote the enrolment of an adequate number of participants, a clinician admitting patients diagnosed with septic shock to intensive care should inform the promoter of the study, the principal investigator of the centre involved, by email.

\section{Enrolment in concurrent studies}

Since this is an observational study, patients who are also included in other observational or experimental studies may be enrolled in this study.

\section{Follow-up procedure}

Telephone contact at 90 days after discharge from the intensive care unit will be carried out to follow up the enrolled patients according to the SF-36 questionnaire.

\section{Statistical plan}

In this study the primary parameter of effectiveness is the evaluation of the percentage of responders .

The analysis will be divided into three panels:

- The first will report the parametric description at baseline and day 2, and the absolute change from baseline of the dose of dobutamine.

- The second will show the parametric description of the change on day 2 from baseline in percentage terms.

- The third will report the percentage of responder patients accompanied by the $95 \%$ CI.

\section{Secondary endpoints}

1. Descriptive analysis with parametric estimates for the following continuous variables:

- GLS.

- CI.

- Ventricular Arterial (VA) coupling.

- Quality of life assessment in the follow-up period evaluated with the SF-36 questionnaire under eight health domains:

- Physical activity.

- Physical and emotional limitations due to clinical condition.

- Physical pain.

- Perception of health status.

- Vitality.

- Social activities.

- Mental health.

- Change in health.

2. Calculation of the survival function, using a KaplanMeier estimator, of the time interval in days from the start of the levosimendan infusion, but only if the number of censored patients is less than $20 \%$, in order to obtain likely estimates of the parameters of the function.

Missing values will be managed using the method of imputation, and unavailable data will be substituted with the mean of values observed.

If the number of patients that can be analysed is close to the sample estimate (see paragraph 1.3), the following descriptive analyses of the stratified endpoints will be performed:

- Gender. male versus female.

- Renal replacement therapy (RRT): RRT versus no RRT.

- Therapy with antiarrhythmic drugs: no versus yes. 
- Race: Caucasian versus whatever else.

- Age: $<65$ years versus $\geq 65$ years.

Descriptive analyses will be prepared to evaluate the degree of correlation between the GLS variable and the heart rate and coupling VA variables.

If the non-responder rate is $>20 \%$, the profile of the responders and those of the non-responders will be compared at a descriptive level.

\section{Patient and public involvement}

Indirect patient and public involvement

We did not directly include patients and the public in this study, but the database used in the study was developed with patient and public involvement (in particular defining the follow-up procedure) and has been updated by a committee that includes patient representatives.

\section{DISCUSSION}

Cardiogenic shock during sepsis can take place with either systolic or diastolic dysfunction; the latter seems to be more frequent in male subjects of young age with high levels of lactate at admission and history of heart failure. ${ }^{20}$ Variations in the ventricular ejection fraction do not appear to be predictors of either sensitive or specific mortality, while strain has been significantly related in some studies to mortality, regardless of the SOFA score. ${ }^{21}$ The development of diastolic dysfunction may also be related to increased mortality, but data in the literature are scarce and conflicting. Among serum biomarkers, high levels of troponin and pro-Brain Natriuretic Peptide (BNP) correlate with increased mortality in sepsis, but the effect disappears when data are stratified, with regard to the severity of the overall clinical picture. ${ }^{22}$

Levosimendan is metabolised in the liver and intestine, and its active metabolite OR-1896, with a half-life of 75-80 hours, is responsible for its continuous effect over a period of 7-9 days after the 24-hour drug infusion. The pharmacokinetic profile of levosimendan is not influenced by gender, race, age or frequent comorbidity in patients with sepsis, such as renal or liver failure. There are no known interactions with drugs commonly used on cardiac patients, such as digoxin, warfarin or beta-blockers. ${ }^{23} 24$

During sepsis, the dephosphorylation of proteins causes a desensitisation of myofibrils against calcium. Levosimendan, with its action of sensitisation to calcium independently of $\beta$-adrenergic receptors, can offer valuable support to dobutamine as an inotropic drug in sepsis. Moreover, due to the absence of effect on intracellular calcium, it does not increase the risk of arrhythmias linked to this alteration of the ion balance nor the overall consumption of oxygen. Acidosis only minimally influences the calcium-sensitising properties of the drug. Levosimendan exerts a moderate inhibitory effect on phosphodiesterase III of the myocardium with increased cyclic adenosine monophosphate (cAMP), resulting in a positive lustre effect. Levosimendan also interacts with different channels of potassium on the plasma membrane of myocytes and mitochondria. Activation of these channels facilitates potassium outflow and promotes cellular hyperpolarisation, which decreases calcium entry into the cells and promotes cell outflow. The net effect is to decrease the concentration of intracellular calcium with consequent vasodilation. ${ }^{12}$

In the light of these encouraging data, a multicentre randomised study in the UK called the LeoPARDS was conducted from 2014 to 2015 to assess whether the addition of levosimendan to routine care in patients with septic shock reduced the severity of organ dysfunction and to assess its safety profile. A total of 516 patients with septic shock who received amine for at least 4 hours were enrolled. The primary objective was the average variation of SOFA. The effect of levosimendan on each apparatus was then analysed, and secondary outcomes were also evaluated, such as days spent without amine infusion, days without mechanical ventilation, the proportion of patients with acute renal failure and the duration of RRT. Finally, mortality at 28 days, days of hospitalisation in intensive care and serious adverse events were also assessed. In this study the addition of levosimendan to routine care did not lead to a decrease in the severity of organ failure in patients with septic shock compared with placebo. Patients treated with levosimendan received higher doses of norepinephrine to maintain the set mean arterial pressure (MAP) values, maintained a higher heart rate and higher incidence of arrhythmias, and were mechanically ventilated for several days. However, as the authors themselves pointed out, this study had many limitations. First, levosimendan was not compared with another inotropic, such as dobutamine, but only with placebo. In both patient groups, MAP was often maintained above the target, so excessive doses of norepinephrine were probably used. Only $30 \%$ of the patients underwent a basic echocardiographic examination and few patients were enrolled with an effectively low CI. Moreover, no echocardiographic examinations were performed to assess any changes in cardiac performance after the administration of levosimendan. For these reasons the authors acknowledged that this trial cannot be a useful guide to choose the best inotropic to use on patients with sepsis with CI changes. ${ }^{25}$ Among the many criticisms raised against this trial, the most common was the lack of a baseline echocardiographic evaluation for the selection of patients with effective septic cardiomyopathy and therefore the absence of identification of patients who really needed inotropic support.

\section{ETHICS AND DISSEMINATION \\ Dissemination statement}

The investigators declare that the study will be conducted in full compliance with international regulations (European Union Directive 2001/20/EC) and with national implementation (Italian ministerial decree 15 July 1997; 211/2003; D. 200/2007) regarding the clinical trial and the principles of the Declaration of Helsinki, in order 
to ensure maximum protection of the subjects involved. The principal investigator shall ensure that the trial is conducted in accordance with this protocol and with GCP. The promoter of the study is committed to the protection of the sensitive personal data, clinical and otherwise, of those involved in the study, as established by national law $(196 / 2003)$.

\section{Data properties}

The ownership of the data, being an independent study in accordance with the Ministerial Decree of 17 December 2004, belongs to the promoter of the study and is shared with the experimenters (Ministerial Decree of 17 December 2004, article 1, paragraph 2, letter c).

\section{Data collection}

The data will be collected on CRF paper, then entered into a central data collection site for both collection and analysis.

\section{Data management}

To eliminate any possible data error, all data will be subjected in advance to an automatic control set; once the problems are solved, the database will be closed and used for statistical surveys.

The statistical surveys will be carried out by an independent statistician.

Before closing the data collection database, a detailed statistical analysis plan (SAP) will be drawn up when the data cleaning activities have been completed on the database.

The SAP will contain a summary of the most relevant aspects of the protocol, the changes that will be implemented with respect to the original analysis plan, the description of any protocol violators/deviators, the profile of the sample and the analyses concerning the clinical variables of effectiveness and safety.

\section{Data retention}

During the course of the study, no information will be entered electronically that would allow a patient to be identified (such as name and/or date of birth). Only the initials and age will be collected and each patient will be assigned a unique identification number, generated by the electronic data collection card; investigators will not be able to access the patient's initials entered in the electronic data collection card. In order to subsequently identify the data entered, each patient will be assigned a unique identification number, called the study ID, when they are entered into the data collection card. In order to facilitate data collection, if the initials are not entered in the database a link note can be created between the initials and the study ID to be maintained only locally. At the end of the study, a verification of the data entered in the database will be carried out. Soon after, the database will be blocked and, before statistical analysis is started, the patient's initials will be deleted and local coordinators will be asked to delete any identification information, including the link between the patient's initials and the study ID. The data will be stored securely and all procedures concerning the management of information will be in line with European Union Data Protection Directives 95/46/EC. After completion of the study, the database will be maintained in such a way as to prevent unauthorised access.

\section{Publication policies}

In accordance with the International Conference on Harmonisation of Technical Requirements for Registration of Pharmaceuticals for Human Use (ICH)-Good Clinicla Practice (GCP), the Principal of the Practice undertakes to produce a report on the Practice, to publish all data collected as described in the protocol and to ensure that the data are reported responsibly and consistently. In particular, the publication of the data resulting from this study will take place independently of the results obtained. The transmission or dissemination of data, through scientific publications and/or presentations in congresses, conferences and seminars and participation in multicentre studies, will take place exclusively following a purely statistical processing of the same, or in any case in absolutely anonymous form. The person responsible for all the research and therefore for the treatment of the data is Dr Iacopo Cappellini.

Contributors $\mathrm{LC}$ is in charge of the study and conceived this protocol. AM drafted this protocol. LC, AM, SC, LZ and MP will collect and manage the data. MP will analyse and interpret the data. LC, AM, SC, LZ, MP and GC wrote this protocol.

Funding Sintesi Research Italy (delegated by Orion Pharma) undertakes to provide the following services to support the study free of charge: data management (creation of electronic databases and CRF management) and statistical processing.

Competing interests This trial has been designed independently of any commercial organisation and will be independently coordinated, managed and analysed.

Patient consent for publication Not required.

Provenance and peer review Not commissioned; externally peer reviewed.

Open access This is an open access article distributed in accordance with the Creative Commons Attribution Non Commercial (CC BY-NC 4.0) license, which permits others to distribute, remix, adapt, build upon this work non-commercially, and license their derivative works on different terms, provided the original work is properly cited, appropriate credit is given, any changes made indicated, and the use is non-commercial. See: http://creativecommons.org/licenses/by-nc/4.0/.

ORCID iD

lacopo Cappellini http://orcid.org/0000-0001-9596-458X

\section{REFERENCES}

1 Howell MD, Davis AM. Management of sepsis and septic shock. JAMA 2017;317:847.

2 Singer M, Deutschman CS, Seymour CW, et al. The third International consensus definitions for sepsis and septic shock (Sepsis-3). JAMA 2016;315:801-10.

3 Beesley SJ, Weber G, Sarge T, et al. Septic cardiomyopathy. Crit Care Med 2018;46:625-34.

4 Lanspa MJ, Pittman JE, Hirshberg EL, et al. Association of left ventricular longitudinal strain with central venous oxygen saturation and serum lactate in patients with early severe sepsis and septic shock. Crit Care 2015;19:304.

5 Dalla K, Hallman C, Bech-Hanssen O, et al. Strain echocardiography identifies impaired longitudinal systolic function in patients with septic shock and preserved ejection fraction. Cardiovasc Ultrasound 2015;13:30. 
$6 \mathrm{Ng}$ PY, Sin WC, Ng AK-Y, et al. Speckle tracking echocardiography in patients with septic shock: a case control study (SPECKSS). Crit Care 2016;20:145

7 Yingchoncharoen T, Agarwal S, Popović ZB, et al. Normal ranges of left ventricular strain: a meta-analysis. J Am Soc Echocardiogr 2013;26:185-91.

8 Nagueh SF, Smiseth OA, Appleton CP, et al. Recommendations for the evaluation of left ventricular diastolic function by echocardiography: an update from the American Society of echocardiography and the European association of cardiovascular imaging. Eur Heart J Cardiovasc Imaging 2016;17:1321-60.

9 Rhodes A, Evans LE, Alhazzani W, et al. Surviving sepsis campaign: international guidelines for management of sepsis and septic shock: 2016. Intensive Care Med 2017;43:304-77.

10 Sato R, Nasu M. Time to re-think the use of dobutamine in sepsis. $J$ Intensive Care 2017;5:65.

11 Matejovic M, Krouzecky A, Radej J, et al. Successful reversal of resistent hypodynamic septic shock with levosimendan. Acta Anaesthesiol Scand 2005;49:127-27.

12 Pinto BB, Rehberg S, Ertmer C, et al. Role of levosimendan in sepsis and septic shock. Curr Opin Anaesthesiol 2008;21:168-77.

13 Morelli A, De Castro S, Teboul J-L, et al. Effects of levosimendan on systemic and regional hemodynamics in septic myocardia depression. Intensive Care Med 2005;31:638-44.

14 Wang Q, Yokoo H, Takashina M, et al. Anti-Inflammatory profile of levosimendan in cecal ligation-induced septic mice and in lipopolysaccharide-stimulated macrophages. Crit Care Med 2015;43:e508-20.

15 Parissis JT, Adamopoulos S, Antoniades C, et al. Effects of levosimendan on circulating pro-inflammatory cytokines and soluble apoptosis mediators in patients with decompensated advanced heart failure. Am J Cardiol 2004;93:1309-12.
16 Grossini E, Molinari C, Pollesello P, et al. Levosimendan protection against kidney ischemia/reperfusion injuries in anesthetized pigs. $J$ Pharmacol Exp Ther 2012;342:376-88.

17 Chen $\mathrm{CH}$, Nakayama M, Nevo E, et al. Coupled systolic-ventricular and vascular stiffening with age: implications for pressure regulation and cardiac reserve in the elderly. J Am Coll Cardiol 1998;32:1221-7.

18 World Medical Association. World Medical association Declaration of Helsinki: ethical principles for medical research involving human subjects. JAMA 2013;310:2191-4.

19 International Conference on Harmonisation of technical requirements for registration of pharmaceuticals for human use. $\mathrm{ICH}$ harmonized tripartite guideline: guideline for good clinical practice. J Postgrad Med 2001;47:45-50.

20 Sato R, Kuriyama A, Takada T, et al. Prevalence and risk factors of sepsis-induced cardiomyopathy: a retrospective cohort study. Medicine 2016;95:e5031.

21 Palmieri V, Innocenti F, Guzzo A, et al. Left ventricular systolic longitudinal function as predictor of outcome in patients with sepsis. Circ Cardiovasc Imaging 2015;8:e003865.

22 Røsjø H, Varpula M, Hagve T-A, et al. Circulating high sensitivity troponin $\mathrm{T}$ in severe sepsis and septic shock: distribution, associated factors, and relation to outcome. Intensive Care Med 2011;37:77-85

23 Gheorghiade M, Teerlink JR, Mebazaa A. Pharmacology of new agents for acute heart failure syndromes. Am J Cardiol 2005;96:68-73.

24 Mebazaa A, Nieminen MS, Filippatos GS, et al. Levosimendan vs. dobutamine: outcomes for acute heart failure patients on betablockers in survive. Eur J Heart Fail 2009;11:304-11.

25 Gordon AC, Perkins GD, Singer M, et al. Levosimendan for the prevention of acute organ dysfunction in sepsis. $N$ Engl J Med 2016;375:1638-48. 